\title{
Improving classification of the external branch of the superior laryngeal nerve with neural monitoring: a research appraisal and narrative review
}

\author{
Yishen Zhao ${ }^{1}$, Zihan Zhao ${ }^{1}$, Daqi Zhang ${ }^{1}$, Yujia Han ${ }^{1}$, Gianlorenzo Dionigi ${ }^{2}$, Hui Sun ${ }^{1}$ \\ ${ }^{1}$ Division of Thyroid Surgery, China-Japan Union Hospital of Jilin University, Jilin Provincial Key Laboratory of Surgical Translational Medicine, \\ Jilin Provincial Precision Medicine Laboratory of Molecular Biology and Translational Medicine on Differentiated Thyroid Carcinoma, Changchun, \\ China; ${ }^{2}$ Division of General Surgery, Head, Endocrine Surgery Section, Istituto Auxologico Italiano IRCCS, Department of Pathophysiology and \\ Transplantation, University of Milan, Milan, Italy \\ Contributions: (I) Conception and design: Y Zhao, Z Zhao; (II) Administrative support: G Dionigi, H Sun; (III) Provision of study materials or \\ patients: H Sun; (IV) Collection and assembly of data: Y Zhao, Z Zhao; (V) Data analysis and interpretation: Y Zhao, Z Zhao; (VI) Manuscript \\ writing: All authors; (VII) Final approval of manuscript: All authors. \\ Correspondence to: Hui Sun. Division of Thyroid Surgery, China-Japan Union Hospital of Jilin University, Jilin Provincial Key Laboratory of Surgical \\ Translational Medicine, Jilin Provincial Precision Medicine Laboratory of Molecular Biology and Translational Medicine on Differentiated Thyroid \\ Carcinoma, Changchun, China. Email: s_h@jlu.edu.cn.
}

\begin{abstract}
Objective: To review the published literature on external branch of superior laryngeal nerve (EBSLN) neural monitoring and propose a new EBSLN classification system using intraoperative neural monitoring (IONM).

Background: The injury rate of the external branch of the superior laryngeal nerve (EBSLN) in thyroid surgery is $0-58 \%$. Symptoms of EBSLN injury are not consistent, and patients often complain of modification of the voice timbre with the preservation of vocal cord function. Standards for the diagnosis of EBSLN injury are lacking.

Methods: The PubMed database was searched using the terms 'External branch of the superior laryngeal nerve' and 'Intraoperative neuromonitoring' from 2010 through March 2020.

Conclusions: This paper reviewed the anatomy of the EBSLN, the diagnosis and treatment of injury, and the application of IONM in the EBSLN. The traditional EBSLN classification method was analyzed and compared with our new classification method. The diagnosis of EBSLN injury is a problem that still needs to be resolved. For anatomic classifications of the EBSLN, we found that the conventional classification systems may not accurately reflect the real status of the EBSLN and the surgical risks that may occur intraoperatively. Using IONM, we developed an EBSLN classification method that was consistent with conventional diagnosis and treatments and can be widely and easily utilized during surgery.
\end{abstract}

Keywords: Thyroid surgery; intraoperative neuromonitoring (IONM); external branch of superior laryngeal nerve (EBSLN); morbidity; anatomical classification

Submitted Jul 16, 2021. Accepted for publication Sep 16, 2021.

doi: $10.21037 / g s-21-518$

View this article at: https://dx.doi.org/10.21037/gs-21-518

\section{Introduction}

This paper aims to review the published literature on external branch of superior laryngeal nerve (EBSLN) neural monitoring and propose a new EBSLN classification system. Until recently, surgeons operating on the thyroid gland paid greater attention to the recurrent laryngeal nerve (RLN) than the EBSLN (1). RLN injury can cause vocal cord paralysis, which may lead to hoarseness, and bilateral injury can appear breathing difficulties, which reduces the patient's quality of life and may even be life-threatening. 
Table 1 List of voice complaints for EBSLN injury

\begin{tabular}{ll}
\hline No. & Voice complaints \\
\hline 1 & Weakness, tightness of the voice, and require extra effort to speak \\
2 & Shortening of the maximum phonation time (especially concerning the sounds /s/, /z/, and /e/) \\
3 & Lowering of the high tone reduction of the vocal range \\
4 & Singing voice may be significantly impaired \\
\hline
\end{tabular}

EBSLN, external branch of superior laryngeal nerve.

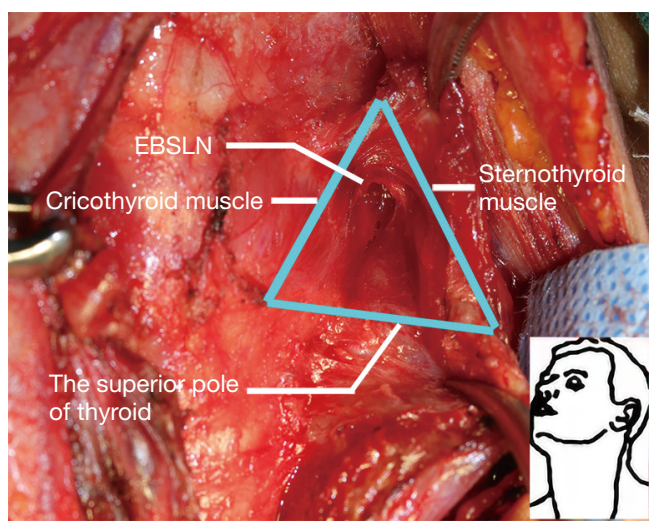

Figure 1 Joll's triangle. EBSLN, external branch of superior laryngeal nerve.

Symptoms of EBSLN injuries are not consistent, and mainly manifest as the fatigue of vocalization and reduction of the sound range It is important for daily life, especially for those who speak in their vocation $(2,3)$ (Table 1). It has also been reported that some female patients are more sensitive to EBSLN injury (3). Litigation in endocrine neck surgery has been limited to cases involving the RLN (4). However, patients with EBSLN injuries often complain of modification of their vocal timbre with the preservation of vocal cord function. Furthermore, standards to accurately diagnose EBSLN injury are lacking (1,5-7).

Due to the different diagnostic methods used in previously published studies, it is difficult to assess the actual prevalence of EBSLN injury (3). Temporary and permanent injury rates of EBSLN are $0-58 \%$ and $3.8 \%$, respectively (5-7). There are numerous reasons for this wide fluctuation of reported rates of injury, including (I) difficulty in measuring EBSLN function (both pre-, intra-, and post-operatively); (II) surgeons' experiences in the identification and dissection of the nerve; (III) a range of diagnostic techniques used in different studies; (IV) use of intraoperative neural monitoring (IONM); (V) the different EBSLN classifications that have been used; (VI) coexisting RLN injury; (VII) intrinsic variability in the anatomy of "Joll's triangle" [for example, the presence of an upper pole thyroid nodule or cancer (Figure 1)]; and (VIII) variations in the surgical approach (i.e., the EBSLN is easier to visualize in the caudal-cranial dissection with the areola endoscopic approach, but is more challenging to visualize in the cranialcaudal dissection used with transoral thyroidectomy) (8). Preservation of the EBSLN during thyroid dissection should be the rule rather than the exception.

Modern thyroid surgery uses three principles to protect the EBSLN: (I) routine identification; (II) IONM navigation within Joll's triangle during dissection; and (III) a meticulous dissection $(2,3,9,10)$.

Compared with the achievements of the predecessors, this article sorts out the new research results of the external branch of the superior laryngeal nerve and intraoperative neuromonitoring. Furthermore, we point out that the protection of EBSLN is weak, and clarifies that the protection of EBSLN is still necessary. In addition, on the basis of Wu's classification, based on the surgical habits of the surgeon, this article proposes the intraoperative EBSLN classification based on IONM technology and the corresponding surgical risk assessment method.

We present the following article in accordance with the Narrative Review reporting checklist (available at https:// dx.doi.org/10.21037/gs-21-518).

\section{Methods}

The PubMed database was searched using the terms 'External branch of the superior laryngeal nerve' and 'Intraoperative neuromonitoring' from 2010 through March 2020.

\section{Discussion}

\section{Surgical anatomy}

Detailed anatomical knowledge of the EBSLN is a key 


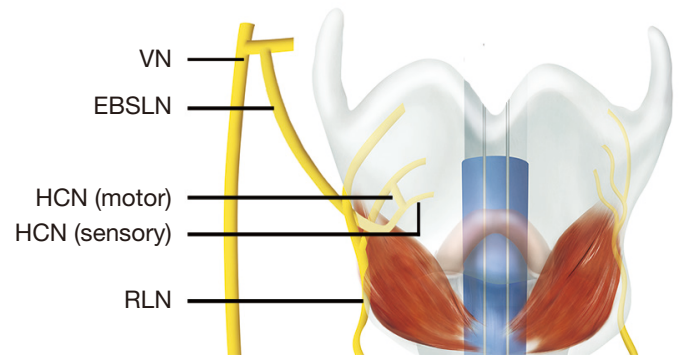

Figure 2 Human communication nerve. HCN, human communication nerve; RLN, recurrent laryngeal nerve; EBSLN, external branch of superior laryngeal nerve; $\mathrm{VN}$, vagus nerve.

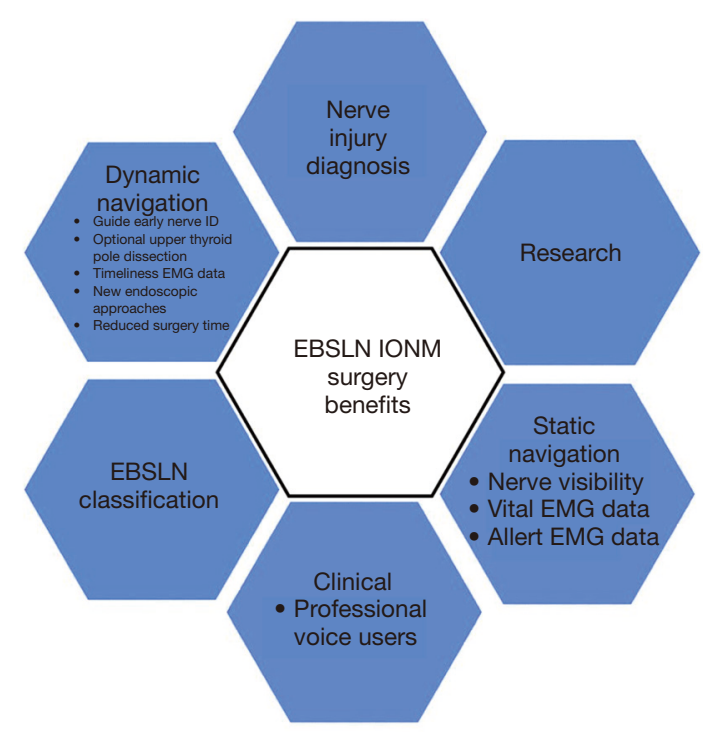

Figure 3 The information of EBSLN IONM is valuable. EBSLN, external branch of superior laryngeal nerve; IONM, intraoperative neural monitoring.

tenet in upper pole thyroid surgical dissection. The superior laryngeal nerve (SLN) branches from the vagus nerve (VN) after it exits the skull. It usually originates from the C2 level (inferior vagal) ganglion near the jugular vein and descends posteriorly to the larynx in the direction of the carotid artery $(3,11,12)$. The SLN passes posterior to the internal carotid artery, through the superior cervical sympathetic ganglion and cervical sympathetic nerve chain, and then descends to the medial part of the thyrohyoid membrane (11). Typically, the SLN is divided into internal and external branches in the superior angle of the hyoid (3). The EBSLN is located on the dorsal side of the carotid sheath, and then crosses medially towards the larynx to innervate the cricothyroid muscle $(3,11)$.
Moosman and DeWeese (13) described the boundaries of the sternothyroid-laryngeal triangle as the sternothyroid muscle above, the thyroid and cricoid cartilages, the pharyngeal constrictors and cricothyroid muscle medially, and the base of the triangle is formed by the superior part the thyroid gland. In this triangular region, the EBSLN is usually located on the medial side, the superior thyroid vein is located on the lateral side, and the superior thyroid artery is positioned between the nerve and vein $(11,13)$. In some cases $(15 \%)$, the EBSLN may adhere to the superior thyroid artery (11). The total length of the EBSLN (which is not affected by gender, race, or left and right sides) is between $31.5 \mathrm{~mm}$ and more than $90 \mathrm{~mm}$ and is approximately $0.2-0.8 \mathrm{~mm}$ wide (3). Maranillo et al. (14) found that approximately $85 \%$ of cases had neural connections between the EBSLN and RLN, which is called the human communication nerve (HCN) (Figure 2).

Technological advances in anatomy: neural monitoring IONM significantly contributed to the understanding of the EBSLN anatomical pathway and its specific localization (Figure 3). Furthermore, during neuromonitoring, it was observed that the waveform could not be recorded in all patients. It has been reported that this unstable monitoring result might be directly related to the lack of a $\mathrm{HCN}$ in some patients (15).

\section{Education}

Classically, principles of EBSLN identification, anatomy, and dissection are taught in the operating room during live endocrine surgeries. Additional educational experiences, such as cadaver dissection and didactic surgical skill tutorials, have been shown to improve confidence and proficiency in residents with respect to both anatomical knowledge and surgical techniques (16). Anatomical simulations in the form of "desktop" three-dimensional (3D) printing are not available for the EBSLN. Thus, IONM provides additional educational opportunities.

\section{Practical approach}

\section{A critical review of EBSLN safety}

Regardless of the approach (open thyroidectomy, endoscopy, robotics, etc.), the critical visualization of Joll's Triangle and the EBSLN must be obtained during upper pole pedicle dissection. The reported visualization rate for the EBSLN is $0-90 \%$ (17). However, mistakes in visual perception are the primary cause of misidentification. In other words, it is easy to mistake the fibers of non-nervous tissue for the EBSLN 


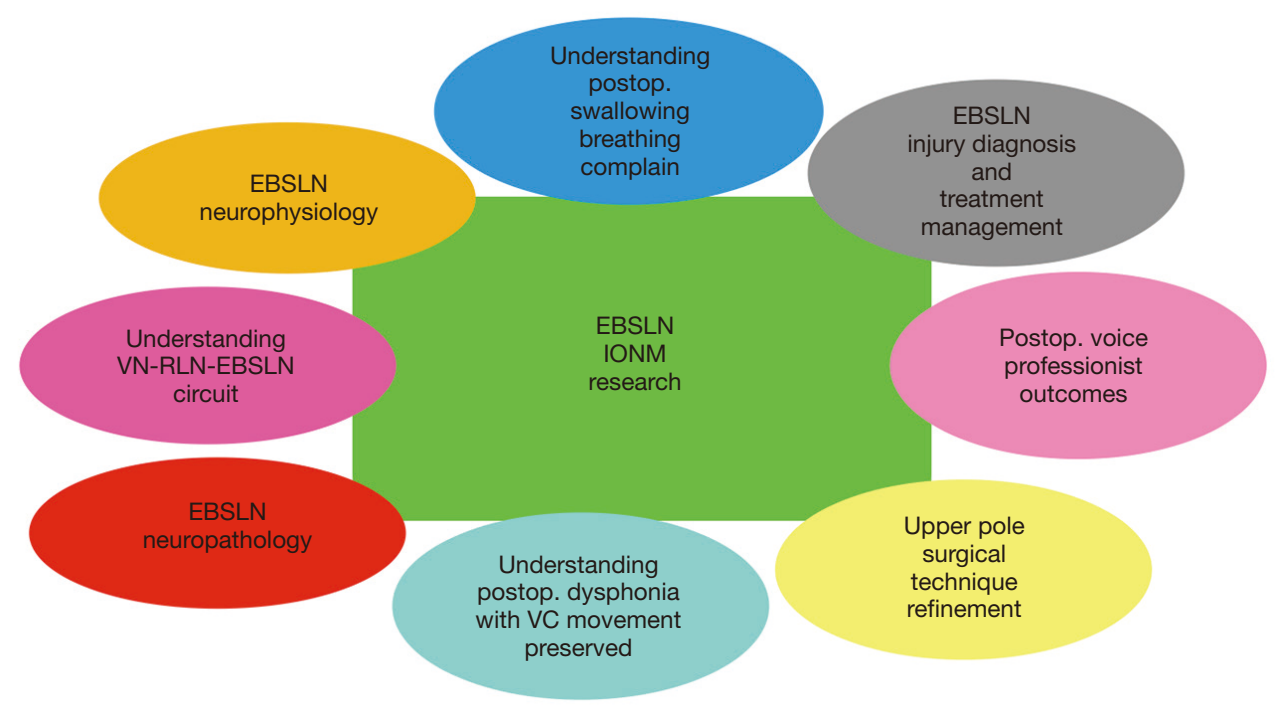

Figure 4 Trends of EBSLN IONM research. EBSLN, external branch of superior laryngeal nerve; IONM, intraoperative neural monitoring.

when applying visual identification to locate and protect this nerve $(9,18)$. The visual misconception of this nerve can be so convincing that endocrine surgeons fail to recognize that the EBSLN has been misidentified, and erroneous transection of the nerve occurs. Visual identification of the EBSLN is difficult, especially in cases where previous surgery, large apical tumors, or bleeding from the upper thyroid pole may impede visualization.

\section{A description of the neural monitoring system}

IONM has been applied to add information to what can be obtained visually and to facilitate the localization and identification of the EBSLN during dissection of the upper pole and ligation during thyroid surgery (Figure 4). IONM has also been used to predict vocal cord function. A recent survey from the International Neuromonitoring Study Group (INMSG) revealed that intermittent IONM was utilized in the management of the RLN by $95 \%$ of 120 respondents. IONM was used to identify the EBSLN by $26.3 \%$ of lowvolume versus $68.4 \%$ of high-volume surgeons $(\mathrm{P}=0.004)$, and $93 \%$ of respondents reported that EBSLN identification using IONM was necessary for voice professionals (19). However, more than $50 \%$ of surgeons had never used IONM for EBSLN identification (19), owing to a lack of experience with proper EBSLN monitoring technology among surgeons. There are several explanations for this: firstly, many surgeons have limited IONM technical experience, such as correctly setting up and using the IONM equipment for EBSLN and understanding the inherent properties of the system to avoid erroneous set-up. Also, many surgeons have inadequate experience in learning how to interpret the results correctly.

The establishment of the EBSLN monitoring system is consistent with the RLN monitoring guidelines (3). Surgery is performed under general anesthesia with naso- or orotracheal intubation using an electromyographic (EMG) tube (Trivantage EMG tube, Medtronic, Jacksonville, Florida, USA) for IONM. Event thresholds need to be set, including a reduced response threshold to identify small responses at $50 \mu \mathrm{V}$, and adjusted according to the specific intraoperative circumstances. EBSLN stimulation is achieved using a monopolar stimulator probe (no. 8225490, Medtronic, Jacksonville, Florida, USA). Invariably, the EBSLN crosses the superior thyroid pole at the bifurcation of the vessels. At this location, the EBSLN is identified as it attaches to the tissue of the superior thyroid pole. According to IONM guidelines, the surgical management of the superior pole should include direct visualization of the EBSLN and a 1 $\mathrm{mA}$ true positive stimulation (i.e., an EMG signal coupled with a cricothyroid muscle twitch). Complementary standardized IONM includes the following: (I) predissection EBSLN mapping and stimulation (S1); (II) stimulation while dissecting the EBSLN (i.e., the superior laryngeal nerve is checked repeatedly at each step of the surgery according to the needs of the surgeon); and (III) final verification of the EBSLN integrity (S2). IONM 
Table 2 The EBSLN identification rate using IONM intraoperatively

\begin{tabular}{lccc}
\hline Author & Year & $\begin{array}{c}\text { Visual } \\
\text { identification (\%) }\end{array}$ & $\begin{array}{c}\text { IONM } \\
\text { identification (\%) }\end{array}$ \\
\hline Loch-Wilkinson (29) & 2007 & 86.0 & 87.0 \\
Dionigi (30) & 2009 & 42.0 & 83.6 \\
Lifante (31) & 2009 & 21.0 & 66.0 \\
Barczyński (32) & 2012 & 34.3 & 83.8 \\
Darr (33) & 2014 & $\mathrm{NR}$ & 100.0 \\
Glover (8) & 2015 & 85.7 & 97.2 \\
Aygün (34) & 2017 & 27.9 & 93.5 \\
Gurleyik (15) & 2018 & 66.9 & 97.1 \\
\hline
\end{tabular}

EBSLN, external branch of superior laryngeal nerve; IONM, intraoperative neural monitoring.

mapping allows for optimization of the level of ligation and section of the branches of the superior thyroid artery while avoiding the EBSLN in the dissected tissue (Video 1).

\section{IONM bighlights}

Application of IONM to the EBSLN enhances several aspects of thyroid surgery, including (I) surgical anatomy, (II) research that increases knowledge of EBSLN neurophysiology and pathology, and (III) refinement of dissection techniques. Another theoretical aspect is that EBSLN monitoring could provide an additional explanation concerning some postoperative consequences in patients, such as dysphonia, and difficulty in breathing and swallowing, which are not expected if only the vagus nerve and the RLN are monitored. EBSLN monitoring could provide important information concerning closure of the VN-RLN-EBSLN circuit and postoperative symptoms that are not associated with vocal cord paralysis.

Barczyński and Lennquist addressed this point using only visual identification, but approximately $20 \%$ of EBSLNs located under the deep fascia of the hypopharyngeal constrictor could not be identified. However, the location could be determined using IONM $(3,20)$. The research of Uludag et al. and the meta-analyses of Naytah et al. and Cheruiyot et al. showed that the application of IONM could reduce the risk of EBSLN injury (21-23).

Snyder and Jonas determined that the use of IONM in the dissection of the superior pole of the thyroid was helpful to measure nerve function $(24,25)$. Gurleyik et al. found that IONM recognized $65.3 \%$ (in the first surgical procedure) and $30.4 \%$ (in subsequent surgical procedures) of the EBSLNs that were identified by visual inspection during surgery, and 55 of the 97 unrecognized nerves were identified by IONM (26). Lee et al. (27) showed that IONM could improve the identification rate of the EBSLN in high-risk surgeries (Cernea type 2B) by $15.9 \%$. Engelsman $e t$ al. reported that the identification rate of the EBSLN using IONM was as high as $98.8 \%$ (28). They also reported that the voice handicap index (VHI) questionnaire survey, which was conducted before and after surgery, did not show any significant changes in score sharing (28). In addition, several researchers have reported the rates of EBSLN identification using IONM, as shown in Table 2. Thus, compared with traditional visual identification, nerve monitoring technology has the advantages of accuracy and early identification, assistance with nerve dissection, localization of subfascial or intramuscular nerves, real-time monitoring, and quantification of nerve function.

\section{EBSLN nomenclature in clinical practice}

The first EBSLN classification system can be traced to a study published by Durham and Harrison in 1964 (35). Currently, Cernea, Kierner, Friedman, and Selvan typing are recognized (Figure 5). At present, Cernea typing is the primary system that is used. There were significant differences in the Cernea typing system reported in different regions and periods $(\mathrm{P}<0.05)$. Based on our literature review, we observed that Cernea typing currently is the main system used in case reports. However, Friedman typing was utilized in a few studies.

\section{Summary of reports of EBSLN classification}

Since the 20th century, EBSLN classification reports have focused primarily on Cernea typing, and significant differences in Cernea typing have been reported in different regions $(\mathrm{P}<0.05)$. From reports, some researchers believe that racial differences of EBSLN classification exist between East and West (36). From March to June 2019 at our institution, 609 cases of thyroidectomy were performed using neuromonitoring, and 731 EBSLNs were evaluated during surgery. We do not usually dissect the hypopharyngeal constrictor muscle to accomplish visual recognition of the nerve, as such dissection presents more disadvantages than advantages, and the use of electrical devices increases the possibility of a loss of protection for the EBSLN due to exposure $(20,37)$. Therefore, we did not employ the strategy of active anatomic exposure of 


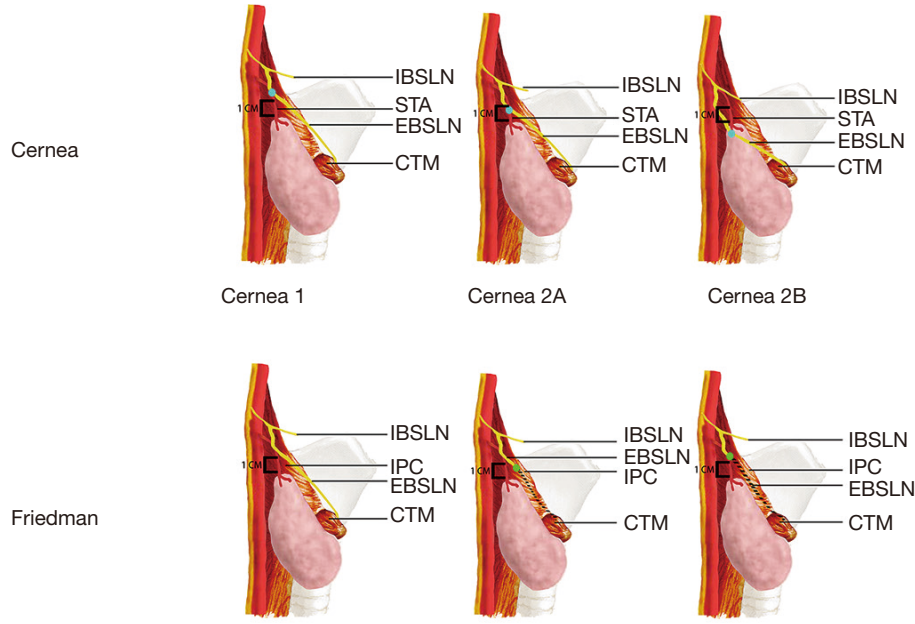

$\begin{array}{lll}\text { Friedman } 1 & \text { Friedman } 2 & \text { Friedman } 3\end{array}$

Kierner

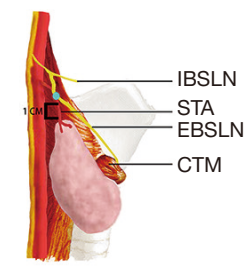

Kierner 1

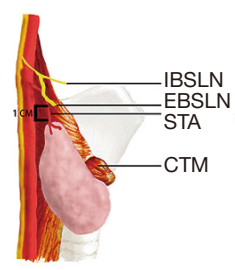

Selvan $1 \mathrm{~A}$

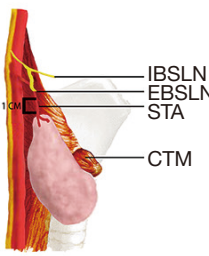

Wu $1 \mathrm{~A}$

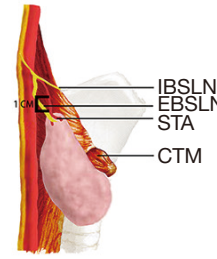

Wu $3 \mathrm{~A}$

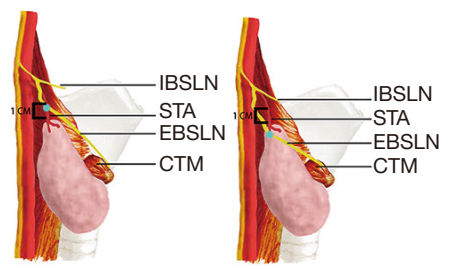

Kierner 3

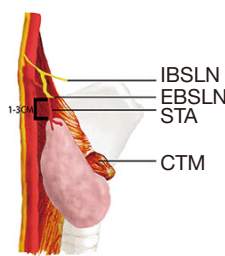

Selvan 2

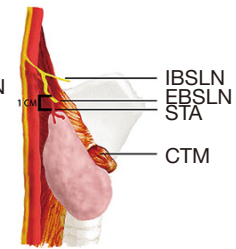

Wu $2 \mathrm{~A}$

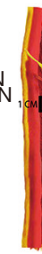

Wu 3B

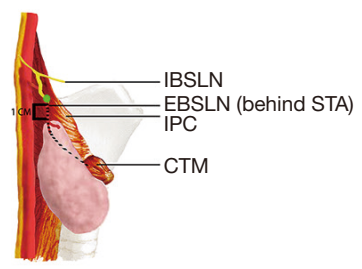

Kierner 4

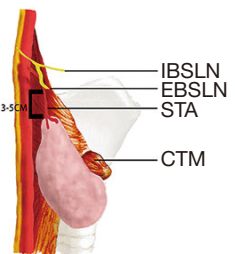

Selvan 3

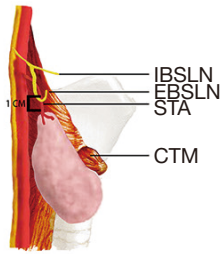

Wu 2B

Figure 5 Classifications of EBSLN. EBSLN, external branch of superior laryngeal nerve. 
the EBSLN during surgery, but only resorted to anatomic exposure of the EBSLN in high-risk cases that combined the course of the nerve with monitoring the EBSLN. The stimulation current was set to $2.0 \mathrm{~mA}$, and the event threshold was set to $50 \mu \mathrm{V}$. Among the 731 EBSLN that were encountered, 54.2\% evoked an EMG signal above the threshold value, and $92.5 \%$ produced a twitch of the cricothyroid muscle after EBSLN stimulation. With respect to classification, Cernea 1 accounted for $79.6 \%$, Cernea 2a accounted for $17.5 \%$, Cernea $2 \mathrm{~b}$ accounted for $2.9 \%, 6.8 \%$ belonged to Friedman 1, 4.9\% belonged to Friedman 2, and

Table 3 EBSLN classification and electric stimulation

\begin{tabular}{ll}
\hline Classification \& stimulation & Rate \\
\hline Cernea & \\
Type 1 & $79.6 \%$ \\
Type 2a & $17.5 \%$ \\
Type 2b & $2.9 \%$ \\
Friedman & \\
Type 1 & $6.8 \%$ \\
Type 2 & $4.9 \%$ \\
Type 3 & $88.3 \%$ \\
Electric stimulation & \\
Get EMG signal & $54.2 \%$ \\
Get cricothyroid muscle twitch & $92.5 \%$ \\
\hline
\end{tabular}

EBSLN, external branch of superior laryngeal nerve.
88.3\% belonged to Friedman 3 (Table 3).

\section{EBSLN injury}

\section{Visualization of the EBSLN only}

EBSLN injury is believed to be the most common and underestimated morbidity following thyroid surgery. For voice-related professionals, EBSLN injuries can be ruinous (2). Cernea and Mangano reported that it was difficult to determine whether or not the EBSLN was damaged during surgery when only visual inspection was used $(38,39)$.

\section{IONM}

Authors have suggested that the best method of IONM to judge the presence and extent of EBSLN injury is to observe and compare the changes in EMG signals collected before (S1) and after treatment of the superior pole vessels (S2), and to observe for a tremor of the cricothyroid muscle induced by EBSLN stimulation at different times intraoperatively (3). If the EBSLN amplitude is decreased or the latency is prolonged during surgery, or the tremor of the cricothyroid muscle that is induced by stimulation of the EBSLN is obviously weakened or disappears, this indicates the possibility of EBSLN injury (3) (Video 1).

\section{Diagnosis of EBSLN damage}

According to the consensus of the American Head and Neck Society, the commonly used methods for diagnosing

Table 4 EBSLN injury diagnosis tools

\begin{tabular}{ll}
\hline Method & Evaluation \\
\hline Indirect (mirror) laryngoscopy & EBSLN injury is extremely difficult to detect by mirror examination \\
$\begin{array}{l}\text { Flexible transnasal fiber-optic } \\
\text { laryngoscopy }\end{array}$ & $\begin{array}{l}\text { Dysfunction of the EBSLN is difficult to diagnose. Testing of cricothyroid muscle EMG may be } \\
\text { required to obtain accurate diagnosis } \\
\text { Laryngeal ultrasound }\end{array}$ \\
Injury of the EBSLN is not well appreciated by transcutaneous laryngeal ultrasound \\
Laryngeal stroboscopy & $\begin{array}{l}\text { Unilateral EBSLN paresis may show vocal fold bowing, vocal fold shortening, and vocal process } \\
\text { height asymmetry where the ipsilateral vocal process overrides the normal contralateral side in }\end{array}$ \\
& $\begin{array}{l}\text { stroboscopic examination. This examination should be avoided when impaired periodicity may } \\
\text { synchronize the strobe light problem }\end{array}$ \\
& $\begin{array}{l}\text { Laryngeal EMG remains a qualitative, not quantitative tool. Complete electrical silence has been } \\
\text { correlated with a poor prognosis for recovery of an immobile vocal cord. The EMG signal depends } \\
\text { on the impedance of the electrode, the position of the electrode in the muscle, and so on. } \\
\text { Quantitative measures still need further research (such as turns analysis) }\end{array}$ \\
\hline
\end{tabular}

EBSLN, external branch of superior laryngeal nerve; EMG, electromyography. 
Table 5 Laryngeal nerve injury characteristics

\begin{tabular}{|c|c|c|}
\hline Laryngeal nerve injury & Symptoms & Clinical features \\
\hline \multirow[t]{2}{*}{ Superior laryngeal nerve } & $\begin{array}{l}\text { Unilateral: paralysis of cricothyroid muscle } \\
\& \text { ipsilateral anesthesia of the larynx } \\
\text { above the vocal cord }\end{array}$ & $\begin{array}{l}\text { (I) Weak voice with decreased pitch; (II) anesthesia of } \\
\text { the larynx on one side; (III) occasional aspiration; (IV) } \\
\text { laryngoscope findings include: (i) askew position of } \\
\text { glottis-ant. Commissure is rotated to healthy side; (ii) } \\
\text { shortening of the VC with loss of tension and VC appears } \\
\text { wavy; (iii) flapping of the paralyzed VC sags down during } \\
\text { inspiration and bulges up during expiration }\end{array}$ \\
\hline & $\begin{array}{l}\text { Bilateral: an uncommon condition; both } \\
\text { the cricothyroid muscles are paralyzed, } \\
\text { along with anesthesia of upper larynx }\end{array}$ & $\begin{array}{l}\text { (I) Paralysis of both VCs; (II) anesthesia of larynx; (III) cough; } \\
\text { (IV) choking fits; (V) weak \& husky voice }\end{array}$ \\
\hline Both (combined/complete) & $\begin{array}{l}\text { Paralysis of all of the muscles of the larynx } \\
\text { on one side except interarytenoid, which } \\
\text { also receives innervation from opposite } \\
\text { side }\end{array}$ & $\begin{array}{l}\text { (I) All the muscles of larynx on one side are paralyzed; (II) VO } \\
\text { lies in the cadaveric position (i.e., } 3.5 \mathrm{~mm} \text { from the midline); } \\
\text { (III) glottic incompetence results in hoarseness of voice and } \\
\text { aspiration of liquid }\end{array}$ \\
\hline
\end{tabular}

EBSLN injury are transnasal laryngoscopy, indirect laryngoscopy, laryngeal ultrasound, and postoperative laryngeal electromyography (Table 4). Among these procedures, only laryngoscopy and postoperative laryngeal electromyography have a relatively good diagnostic ability for the EBSLN (40).

It also is important to note the difficulty of differentiating and diagnosing unilateral or bilateral paralysis of the EBSLN (Table 5).

\section{Treatment of EBSLN injury}

It is important to note that not only is it difficult to diagnose a nerve injury, but the management of the nerve injury also is not standardized. The same therapy for recurrent laryngeal nerve palsy is now being used for alleged lesions of the upper laryngeal nerve (i.e., speech therapy). Voice therapy is the most commonly prescribed treatment for long-standing, isolated EBSLN paralysis, although improvement in vocal abilities varies. Therapy is targeted at building cricothyroid muscle strength using activities such as glissando maneuvers (41). However, no studies have been published that adequately evaluate the effects of voice therapy on EBSLN paralysis.

Although not commonly performed, there are several small case series describing surgical treatment for EBSLN injury. One study by Shaw et al. (42) used carefully applied unilateral cricothyroid approximation in a series of 10 patients and reported excellent improvement in vocal quality and range. A long-term follow-up for a similar procedure revealed that persistent improvement in quality decreased in range over time, which was likely due to decreased tension on the sutures over time. Nasseri and Maragos (43) performed both type 1 and type 4 thyroplasty in nine patients, and achieved good results. However, more research needs to be performed to prove that these surgeries are effective.

\section{Classification of EBSLN under IONM}

According to the statistics described above, the rate of recognition of the EBSLN under neural monitoring can reach more than $90 \%$. Approximately $20 \%$ of the EBSLNs that are located under the deep fascia of hypopharyngeal constrictor cannot be recognized, but these nerves can be assessed using IONM. For patients who are at high risk of nerve injury and present difficulties in visual recognition of the EBSLN, especially after additional surgery, IONM technology can significantly improve the rate of neural recognition. It is challenging to evaluate nerve function during surgery using only visual inspection, but IONM can provide intraoperative function measurement through intraoperative EMG signals and observation of cricothyroid twitches.

\section{Analysis of our EBSLN classification data}

The Cernea typing results reported by our institution are similar to those reported by Whitfield et al. (44); $80 \%$ of the 


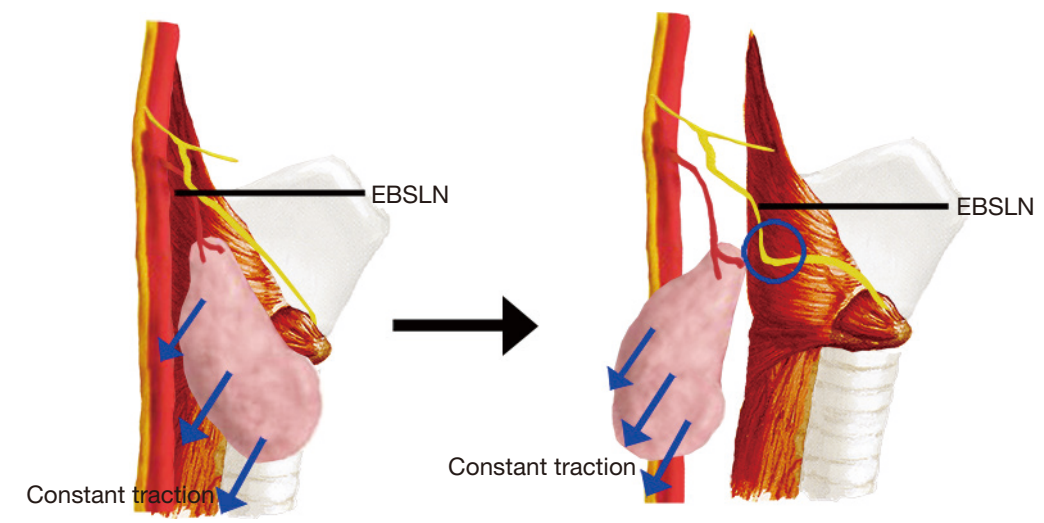

Figure 6 EBSLN shows a "V" shape when outward and downward traction was applied to the nerve. EBSLN, external branch of superior laryngeal nerve.

patients were identified as Cernea type 1 using IONM. The length of the SLN and its branches were not influenced by race, gender, and left or right location (3). Therefore, this result is not due to differences in race. The variations we observed from the mainstream report might be due to the higher proportion of small thyroids that were encountered. Cernea reported that the Cernea type 1 classification occurred in approximately $68 \%$ of cases with small thyroid glands (10).

Some scholars believe that over-dissection of the hypopharyngeal constrictor to expose the EBSLN increases the extent of the surgery and the possibility for greater damage, but also specifically increases the risk of damage to the EBSLN $(20,36)$. Our institution did not endorse a strategy of active anatomic exposure of the EBSLN during every surgery. Instead, the recommendation was to anatomically expose the EBSLN only in high-risk cases, in combination with following the course of the external branch of the superior laryngeal nerve and use of nerve monitoring. Thus, monitoring EBSLNs that were closely related to the superior pole blood vessels and thyroid revealed that the EBSLN classifications were Cernea types $2 \mathrm{a}$ and $2 \mathrm{~b}$. According to the data, the vast majority of EBSLNs are not recognized through visual inspection during surgery. Most of the nerves can be placed into one of the three Friedman classifications. Gavid et al. (45) reported that approximately 50\% (of 144 patients) of the EBSLNs were classified as Friedman type 1, and the remaining nerves were classified as Friedman type 2 (25\%) and Friedman type 3 (25\%). We observed that classification of EBSLNs as Friedman type 1 and type 2 was rare in our institution within the scope of conventional surgery and without the goal of precise anatomical recognition. This result emphasized the importance of the use of IONM in thyroid surgery. Given that there were numerous cases in which it is not possible to recognize the EBSLN visually, it was advantageous to ensure recognition of the EBSLN through the use of neural monitoring to allow for a smaller surgical area and avoid over-dissection of the pharyngeal constrictor. An effective EMG of the EBSLM could not be obtained for approximately $50 \%$ of the patients at our institution. However, $92.5 \%$ of EBSLNs were located through nerve stimulation that resulted in a tremor of the cricothyroid muscle. Surgeries in patients who failed to exhibit cricothyroid tremors usually went deeper because of the EBSLN, but this was not related to the surgical area.

\section{A new EBSLN classification system, Sun's classification} The Cernea and Friedman systems of classification can accurately reflect the trajectory and risk of the EBSLN without surgical intervention or autopsy. However, when dissecting the thyroid superior pole region, there are several situations that place the EBSLN at a high risk of injury, including the following: (I) the presence of goiter that is greater than $100 \mathrm{~g}$, or the upper edge of the thyroid gland is higher; (II) a thyroid gland that weighs more than $240 \mathrm{~g}$; (III) a very large mass located on the thyroid or a short, thick neck; (IV) the need for thyroid cancer surgery; and (V) a second surgery is needed that results in unclear anatomical relationships or severe adhesions of surrounding tissues. In the situations described above, it is usually necessary to pull the thyroid gland down and out to obtain a better surgical field, and this manipulation might change the course of the EBSLN (Figure 6). 

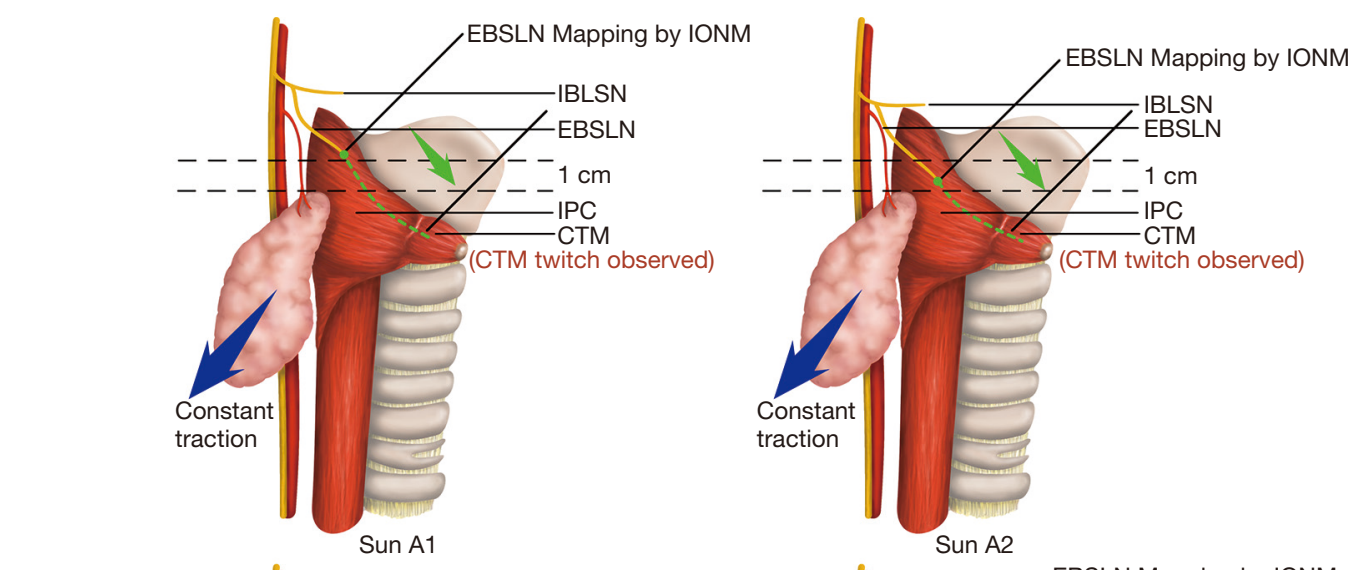

Sun

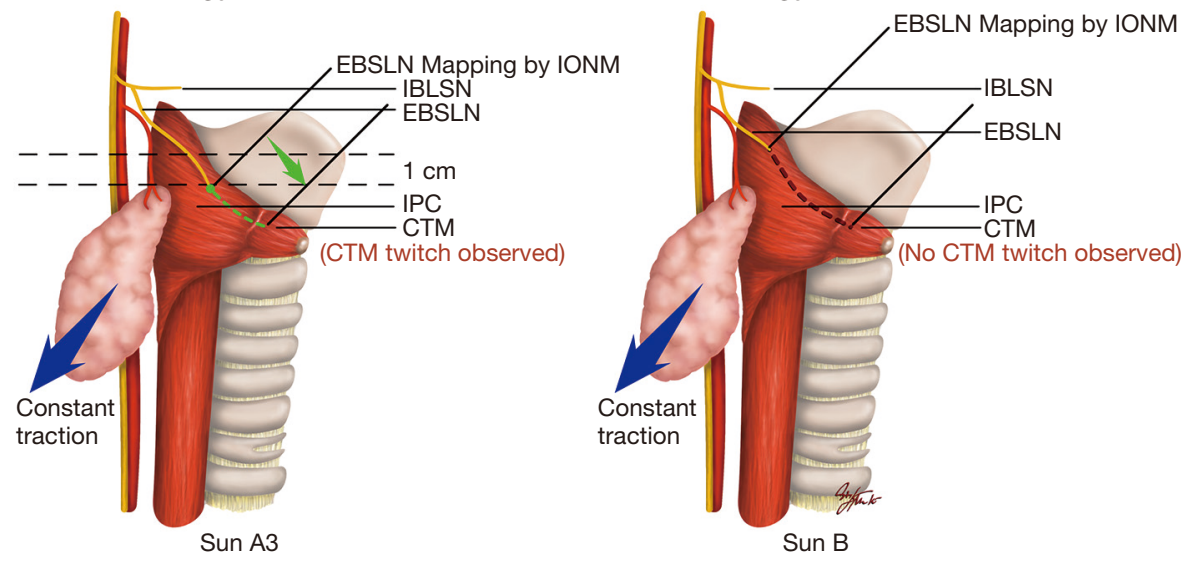

Figure 7 Sun's classification.

Before dissection of the EBSLN, according to published guidelines 1, the EBSLN course that is mapped using small current stimulation was also affected by the outward and downward traction on the thyroid gland, which reveals a "V" shape (Figure 7). Based on the situation described above, we proposed a new EBSLN classification scheme, which is based on the anatomical structure of the superior pole of the thyroid during surgery. It detects and maps the EBSLN using IONM only after pulling the thyroid gland downward or laterally downward, instead of first locating the EBSLN using IONM. The classification can be divided into types $\mathrm{A}$ and $\mathrm{B}$, according to the presence or absence of a twitch of the cricothyroid muscle that is evoked by a small current $(0.5-1 \mathrm{~mA})$ stimulation. The type A classification is further divided into three subgroups according to the distance between the intersection of the blood vessels located at the superior thyroid pole, detection of the EBSLN using neural monitoring, and the anatomic plane of the superior thyroid pole. The distance of type 1 is longer than $1 \mathrm{~cm}$, the distance for type 2 is $0-1 \mathrm{~cm}$, and the distance for type 3 is below the plane of the superior pole of the thyroid (Table 6).

EBSLN classified as type A2 and A3 have a higher risk of injury. Therefore, we recommend anatomically exposing the EBSLN in cases classified as A2 and A3, as well as paying particular attention to avoid direct and lateral thermal injury to the EBSLN when using electrical devices to treat the blood vessels located at the upper pole of the thyroid. We also recommend that type A1 EBSLNs not be dissected. For type B EBSLNs, we also recommend not to anatomically expose the EBSLN after increasing the stimulation current.

Wang et al. (46) proposed Wu's classification that was based on the anatomy of EBSLN, as observed during surgery. Wang et al. classified the EBSLN based on where it entered into the pharyngeal constrictor, or the lowest level of the EBSLN while under external downward traction. Next, the corresponding risks of different EBSLN classifications were defined from a surgical perspective. Since Wu's classification standard was based on the lowest point of the EBSLN at which it entered the muscle or the 
Table 6 The new anatomic classification of EBSLN

\begin{tabular}{ll}
\hline Type & Method \\
\hline A1 & $\begin{array}{l}\text { Get cricothyroid muscle twitch; IONM indicated that the nerve crossed the superior thyroid vessels } 1 \mathrm{~cm} \\
\text { (or more) above a horizontal plane passing the upper border of the superior thyroid pole }\end{array}$ \\
A2 & $\begin{array}{l}\text { Get cricothyroid muscle twitch; IONM indicated that the nerve crosses the superior thyroid vessels }<1 \mathrm{~cm} \\
\text { above a horizontal plane passing the upper border of the superior thyroid pole }\end{array}$ \\
A3 & $\begin{array}{l}\text { Get cricothyroid muscle twitch; IONM indicated that the nerve crosses the superior thyroid vessels under a } \\
\text { horizontal plane passing the upper border of the superior thyroid pole }\end{array}$ \\
B & Cannot get cricothyroid muscle twitch
\end{tabular}

EBSLN, external branch of superior laryngeal nerve; IONM, intraoperative neural monitoring.

Table 7 The advantages of Sun's classification

\begin{tabular}{ll}
\hline No. & Advantages \\
\hline 1 & Reducing the misclassification caused by EBSLN traction during thyroid surgery \\
2 & Reducing unnecessary exposure of EBSLN, decreasing intraoperative nerve injury and postoperative complications \\
3 & $\begin{array}{l}\text { EMG assisted classification to reduce the improper choice of surgical strategy caused by subjective judgment (such as } \\
\text { visual recognition) }\end{array}$ \\
\hline
\end{tabular}

EBSLN, external branch of superior laryngeal nerve; IONM, intraoperative neural monitoring.

lowest point of the EBSLN that was observed to complete the intraoperative classification, more extensive surgical area and more detailed surgeries were needed. Therefore, this classification system presents difficulties in practical application.

Sun's classification method advocates no need for direct dissection of the EBSLN. Firstly, small current stimulation is used to precisely locate the EBSLN. A decision is then made as to whether to dissect the EBSLN, in order to minimize possible injury to the anterior cervical banded muscle and EBSLN due to the surgery. However, Sun's typing method has the following limitations: (I) the ability to find the EBSLN using low current requires a learning curve, and the operator needs to be proficient in troubleshooting IONM; and (II) a few variant EBSLNs will turn back and cross the superior blood vessel twice. This increases the chance of injuring the EBSLN if the superior pole blood vessel is directly coagulated based on this typing method. The operator needs to locate the EBSLN accurately using a small current stimulation electrode and develop a surgical strategy based on the actual situation (Table 7).

This new classification has not been widely promoted in clinic. The purpose of this article is to present this method to researchers. We hope to accept the review and criticism of experts, and jointly promote the development of EBSLN intraoperative protection and thyroid surgery techniques.

\section{Conclusions}

Although it is generally believed that EBSLN injuries are typically relatively minor, it is catastrophic for specific individuals who use their voices professionally. The conventional method that uses visual recognition can rarely accurately assess EBSLN injury intraoperatively. IONM has been used to compare the changes of myoelectric signals collected before and after the treatment of superior pole vessels and the cricothyroid muscle twitch induced by the stimulated EBSLN in different surgical protocols and procedures. If the EBSLN amplitude decreased significantly or the latency became prolonged during the surgery, or if the cricothyroid muscle twitch induced by EBSLN stimulation disappears, this indicates the possibility of EBSLN injury. Although the application of stroboscopic laryngoscopy to observe vocal cord movement can be used to assess EBSLN integrity and function, research shows that this technology still needs to be standardized, and the diagnosis of EBSLN injury is still insufficient. 
Other postoperative auxiliary diagnostic methods, such as Voice Handicap Index 10 (VHI 10), the grade, roughness breathiness, asthenia, and strain (GRBAS) scale, voice analysis software, and others, only analyze the influence of the voice on life, voice conditions, and voice-related parameters. These methods cannot directly and accurately diagnose the voice problems of patients that are due to EBSLN injury. In summary, the diagnosis of EBSLN injuries is a problem that still needs to be resolved.

For anatomic classifications of the EBSLN, we found that the conventional classification systems may not accurately reflect the real status of the EBSLN and surgical risks that may occur intraoperatively. The Sun's classification method that we advocate is based on the research methods from surgeons' and studies that took place during actual surgeries. With the help of IONM, we developed an EBSLN classification method that was consistent with conventional diagnosis and treatments, and can be widely and easily used during surgery.

However, several factors limited our research. Since all the data were collected from patients, it was not possible to thoroughly dissect the EBSLNs to definitively assess the actual anatomical types of the EBSLNs and their differences during surgery. Our analysis lacked definitive proof of EBSLN damage, which limited our assessments. Based on our observations, it appeared that the intraoperative visual recognition of the EBSLN in a Chinese population might not be commensurate to what has been described in other international reports.

Even with the assistance of neural monitoring technology, the vast majority of EBSLNs are not visible during surgical procedures. Thus, the application of IONM to protect the EBSLN during surgery is highly significant. IONM can effectively reduce unnecessary dissection and improve the surgeon's ability to recognize the EBSLN and its pathway.

\section{Acknowledgments}

Funding: None.

\section{Footnote}

Reporting Checklist: The authors have completed the Narrative Review reporting checklist. Available at https:// dx.doi.org/10.21037/gs-21-518

Conflicts of Interest: All authors have completed the ICMJE uniform disclosure form (available at https://dx.doi. org/10.21037/gs-21-518). GD serves as an unpaid editorial board member of Gland Surgery from Dec 2016 to Nov 2022. The other authors have no conflicts of interest to declare.

Ethical Statement: The authors are accountable for all aspects of the work in ensuring that questions related to the accuracy or integrity of any part of the work are appropriately investigated and resolved.

Open Access Statement: This is an Open Access article distributed in accordance with the Creative Commons Attribution-NonCommercial-NoDerivs 4.0 International License (CC BY-NC-ND 4.0), which permits the noncommercial replication and distribution of the article with the strict proviso that no changes or edits are made and the original work is properly cited (including links to both the formal publication through the relevant DOI and the license). See: https://creativecommons.org/ licenses/by-nc-nd/4.0/.

\section{References}

1. Delbridge L. The 'neglected' nerve in thyroid surgery: the case for routine identification of the external laryngeal nerve. ANZ J Surg 2001;71:199.

2. Friedman M, LoSavio P, Ibrahim H. Superior laryngeal nerve identification and preservation in thyroidectomy. Arch Otolaryngol Head Neck Surg 2002;128:296-303.

3. Barczyński M, Randolph GW, Cernea CR, et al. External branch of the superior laryngeal nerve monitoring during thyroid and parathyroid surgery: International Neural Monitoring Study Group standards guideline statement. Laryngoscope 2013;123 Suppl 4:S1-14.

4. Dralle H, Lorenz K, Machens A. Verdicts on malpractice claims after thyroid surgery: emerging trends and future directions. Head Neck 2012;34:1591-6.

5. Bevan K, Griffiths MV, Morgan MH. Cricothyroid muscle paralysis: its recognition and diagnosis. J Laryngol Otol 1989;103:191-5.

6. Jansson S, Tisell LE, Hagne I, et al. Partial superior laryngeal nerve (SLN) lesions before and after thyroid surgery. World J Surg 1988;12:522-7.

7. Teitelbaum BJ, Wenig BL. Superior laryngeal nerve injury from thyroid surgery. Head Neck 1995;17:36-40.

8. Glover AR, Norlén O, Gundara JS, et al. Use of the Nerve Integrity Monitor during Thyroid Surgery Aids 
Identification of the External Branch of the Superior Laryngeal Nerve. Ann Surg Oncol 2015;22:1768-73.

9. Selvan B, Babu S, Paul MJ, et al. Mapping the compound muscle action potentials of cricothyroid muscle using electromyography in thyroid operations: a novel method to clinically type the external branch of the superior laryngeal nerve. Ann Surg 2009;250:293-300.

10. Cernea CR, Ferraz AR, Nishio S, et al. Surgical anatomy of the external branch of the superior laryngeal nerve. Head Neck 1992;14:380-3.

11. Kochilas X, Bibas A, Xenellis J, et al. Surgical anatomy of the external branch of the superior laryngeal nerve and its clinical significance in head and neck surgery. Clin Anat 2008;21:99-105.

12. Kierner AC, Aigner M, Burian M. The external branch of the superior laryngeal nerve: its topographical anatomy as related to surgery of the neck. Arch Otolaryngol Head Neck Surg 1998;124:301-3.

13. Moosman DA, DeWeese MS. The external laryngeal nerve as related to thyroidectomy. Surg Gynecol Obstet 1968;127:1011-6.

14. Maranillo E, León X, Quer M, et al. Is the external laryngeal nerve an exclusively motor nerve? The cricothyroid connection branch. Laryngoscope 2003;113:525-9.

15. Gurleyik E, Gurleyik G. Intraoperative Monitoring of External Branch of the Superior Laryngeal Nerve: Functional Identification, Motor Integrity, and its Role on Vocal Cord Function. J Invest Surg 2018;31:509-14.

16. Dionigi G, Kim HY, Randolph GW, et al. Prospective validation study of Cernea classification for predicting EMG alterations of the external branch of the superior laryngeal nerve. Surg Today 2016;46:785-91.

17. Prades JM, Lelonge Y, Farizon B, et al. Intraoperative neuromonitoring by vagus nerve stimulation in thyroid surgery: Clinical assessment of recurrent and superior laryngeal nerves. Eur Ann Otorhinolaryngol Head Neck Dis 2020;137:227-30.

18. Aina EN, Hisham AN. External laryngeal nerve in thyroid surgery: recognition and surgical implications. ANZ J Surg 2001;71:212-4.

19. Barczyński M, Randolph GW, Cernea C, et al. International survey on the identification and neural monitoring of the EBSLN during thyroidectomy. Laryngoscope 2016;126:285-91.

20. Lennquist S, Cahlin C, Smeds S. The superior laryngeal nerve in thyroid surgery. Surgery 1987;102:999-1008.

21. Uludag M, Aygun N, Kartal K, et al. Contribution of intraoperative neural monitoring to preservation of the external branch of the superior laryngeal nerve: a randomized prospective clinical trial. Langenbecks Arch Surg 2017;402:965-76.

22. Naytah M, Ibrahim I, da Silva S. Importance of incorporating intraoperative neuromonitoring of the external branch of the superior laryngeal nerve in thyroidectomy: A review and meta-analysis study. Head Neck 2019;41:2034-41.

23. Cheruiyot I, Kipkorir V, Henry BM, et al. Surgical anatomy of the external branch of the superior laryngeal nerve: a systematic review and meta-analysis. Langenbecks Arch Surg 2018;403:811-23.

24. Jonas J, Bähr R. Neuromonitoring of the external branch of the superior laryngeal nerve during thyroid surgery. Am J Surg 2000;179:234-6.

25. Snyder SK, Sigmond BR, Lairmore TC, et al. The longterm impact of routine intraoperative nerve monitoring during thyroid and parathyroid surgery. Surgery 2013;154:704-11; discussion 711-3.

26. Gurleyik E, Dogan S, Cetin F, et al. Visual and electrophysiological identification of the external branch of superior laryngeal nerve in redo thyroid surgery compared with primary thyroid surgery. Ann Surg Treat Res 2019;96:269-74.

27. Lee J, Fraser S, Glover A, et al. Prospective evaluation of the utility of routine neuromonitoring for an established thyroid surgical practice. ANZ J Surg 2017;87:E138-42.

28. Engelsman AF, Warhurst S, Fraser S, et al. Influence of neural monitoring during thyroid surgery on nerve integrity and postoperative vocal function. BJS Open 2018;2:135-41.

29. Loch-Wilkinson TJ, Stalberg PL, Sidhu SB, et al. Nerve stimulation in thyroid surgery: is it really useful? ANZ J Surg 2007;77:377-80.

30. Dionigi G, Boni L, Rovera F, et al. Neuromonitoring and video-assisted thyroidectomy: a prospective, randomized case-control evaluation. Surg Endosc 2009;23:996-1003.

31. Lifante JC, McGill J, Murry T, et al. A prospective, randomized trial of nerve monitoring of the external branch of the superior laryngeal nerve during thyroidectomy under local/regional anesthesia and IV sedation. Surgery 2009;146:1167-73.

32. Barczyński M, Konturek A, Stopa M, et al. Randomized controlled trial of visualization versus neuromonitoring of the external branch of the superior laryngeal nerve during thyroidectomy. World J Surg 2012;36:1340-7.

33. Darr EA, Tufano RP, Ozdemir S, et al. Superior laryngeal 
nerve quantitative intraoperative monitoring is possible in all thyroid surgeries. Laryngoscope 2014;124:1035-41.

34. Aygün N, Uludağ M, İşgör A. Contribution of intraoperative neuromonitoring to the identification of the external branch of superior laryngeal nerve. Turk J Surg 2017;33:169-74.

35. Durham CF, Harrison TS. The surgical anatomy of the superior laryngeal nerve. Surg Gynecol Obstet 1964;118:38-44.

36. Hwang SB, Lee HY, Kim WY, et al. The anatomy of the external branch of the superior laryngeal nerve in Koreans. Asian J Surg 2013;36:13-9.

37. Dionigi G. Energy based devices and recurrent laryngeal nerve injury: the need for safer instruments. Langenbecks Arch Surg 2009;394:579-80; author reply 581-6.

38. Cernea CR, Ferraz AR, Furlani J, et al. Identification of the external branch of the superior laryngeal nerve during thyroidectomy. Am J Surg 1992;164:634-9.

39. Mangano A, Lianos GD, Boni L, et al. Intraoperative neuromonitoring of the external branch of the superior laryngeal nerve during thyroidectomy: the need for evidence-based data and perioperative technical/ technological standardization. ScientificWorldJournal 2014;2014:692365.

40. Sinclair CF, Bumpous JM, Haugen BR, et al. Laryngeal

Cite this article as: Zhao Y, Zhao Z, Zhang D, Han Y, Dionigi G, Sun H. Improving classification of the external branch of the superior laryngeal nerve with neural monitoring: a research appraisal and narrative review. Gland Surg 2021;10(9):28472860. doi: $10.21037 /$ gs-21-518 examination in thyroid and parathyroid surgery: An American Head and Neck Society consensus statement: AHNS Consensus Statement. Head Neck 2016;38:811-9.

41. Dursun G, Sataloff RT, Spiegel JR, et al. Superior laryngeal nerve paresis and paralysis. J Voice 1996;10:206-11.

42. Shaw GY, Searl JP, Hoover LA. Diagnosis and treatment of unilateral cricothyroid muscle paralysis with a modified Isshiki type 4 thyroplasty. Otolaryngol Head Neck Surg 1995;113:679-88.

43. Nasseri SS, Maragos NE. Combination thyroplasty and the "twisted larynx:" combined type IV and type I thyroplasty for superior laryngeal nerve weakness. J Voice 2000;14:104-11.

44. Whitfield P, Morton RP, Al-Ali S. Surgical anatomy of the external branch of the superior laryngeal nerve. ANZ J Surg 2010;80:813-6.

45. Gavid M, Dubois MD, Larivé E, et al. Superior laryngeal nerve in thyroid surgery: anatomical identification and monitoring. Eur Arch Otorhinolaryngol 2017;274:3519-26.

46. Wang K, Cai H, Kong D, et al. The Identification, Preservation and Classification of the External Branch of the Superior Laryngeal Nerve in Thyroidectomy. World J Surg 2017;41:2521-9.

(English Language Editor: A. Kassem) 